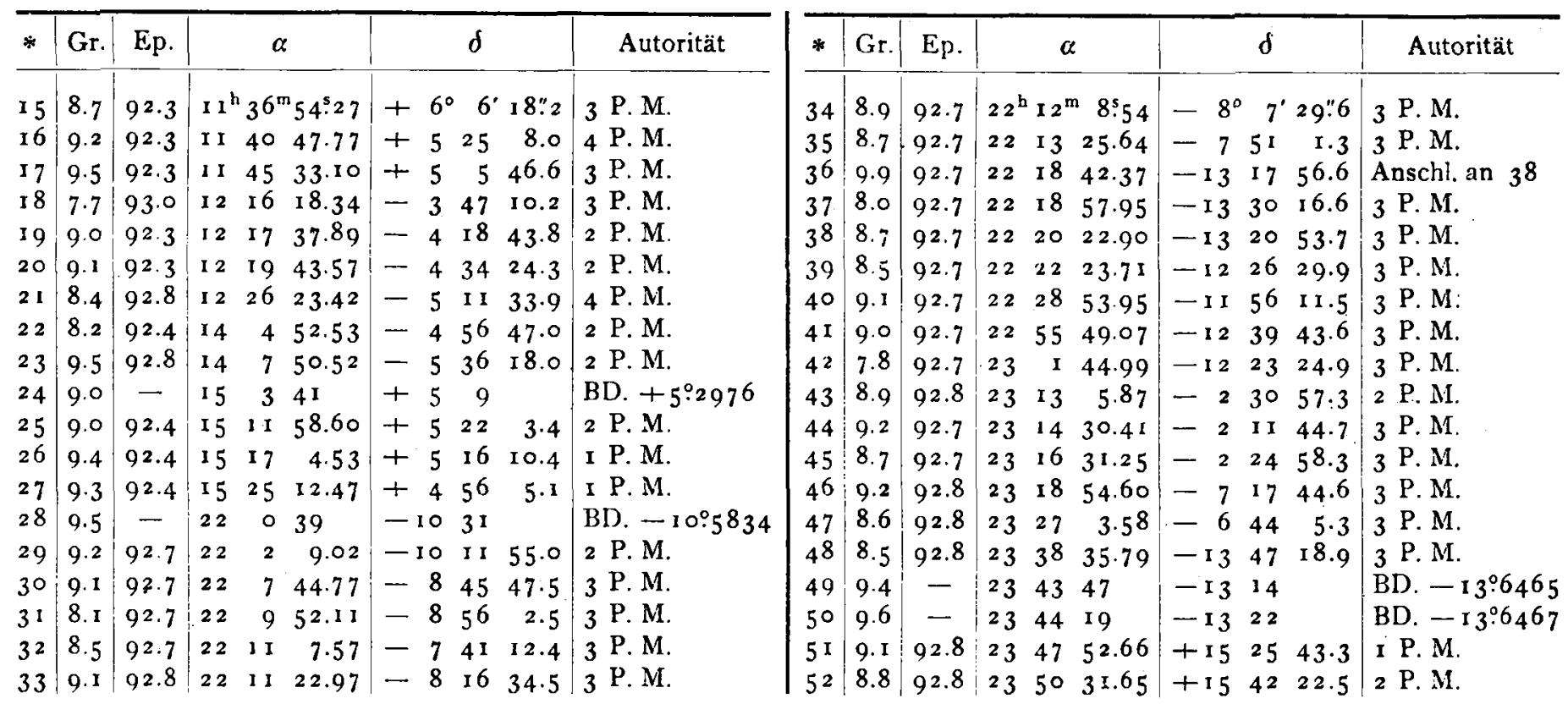

Die Planeten Hebe, Antiope, Sibylla, Idunna und Germania sind am 30 Zöller beobachtet, die übrigen am I 5 Zöller.

Die Vergleichsterne sind am hiesigen Meridiankreise von Herrn Romberg beobachtet worden.

Die fehlenden Sternpositionen, sowie die Sterne, welche nur einmal beobachtet sind, werden bei nächster Gelegenheit gegeben werden. Benerkungen.

Hebe. Mai 1 2. Wolken. - Mai r9, 20. Nebel. - $\quad$ Meliboea. Oct. 11. Leichte Wolken. Mai 27, Juni 7 . Sehr schlechte Bilder.

Nysa. Aug. 26. Starker Wind. - Aug. 30. Nebel. - Sept. 7. Die Nähe des Mondes stört.

Antiope. März 31, Apr. 4. Sehr schlechte Bilder.

A gle. Sept. 30. Sehr schlechte Bilder.

Klotho. Aug. 24. Wolken. - Aug. 26. Starker Wind. - Aug. 29. Starker Wind und sehr schlechte Bilder. Sept. 7. Der Mond sehr nahe.

Juewa. Sept. 1. Starker Wind. - Sept. 9. Der Mond sehr nahe.

Idunna. Apr. 27. Nebel.

Philomela. Sept. I5, 16. Nebel.

Germania. März r. Sehr schlechte Bilder.

Eukrate. Oct. 7. Der Mond sehr nahe. Wolken. - Oct. 19. Leichte Wolken.

Pulkowo 1893 Mai 3 .

W. Seraphimoff.

\title{
Observations of Comet 1892 VI at Windsor, N. S. Wales.
}

\section{By Fohn Tebbutt.}

I herewith transmit to you my complete observations of this comet. They were all made with a square barmicrometer in a dark field, the $4 \frac{1}{2}$ inch equatorial being employed on the first two dates and the 8 inch instrument on all the remaining dates. In November and December the comet had a fairly bright condensation. Subsequently this condensation grew fainter but at the same time smaller, and its gradual diminution facilitated observation at the edges of the micrometer bars. A faint tail was occasionally perceptible in the telescope during November, December and January. Owing to cloud or haze observations were unsatisfactory on December 20,21 , January 7,25 , February 28 , March 13, 20, 24, 26; April 19, 21, 23. The comparison star of January 13 was double, and its observation therefore difficult; the brighter component was employed. The observations of February 14, March 20, April 17 , in consequence of the proximity of small stars, were made with difficulty. In the first seven comparisons of the last mentioned date the comet gradually approached a star of the $8^{\text {th }}$ magnitude and in the last three comparisons both objects were observed as one. On this date too the comet and a small nebula somewhat brighter than itself could just be distinguished in the finder of 2.4 inches aperture by looking obliquely into the eyepiece. On April 20 the comet was seen as a faint condensed point, with scarcely any surrounding coma. After the withdrawal of the moon the comet was picked up on April 13 by extending Dr. Ristenpart's ephemeris in A. N. $3^{1} \mathbf{3}^{1}$. His extended epherneris did not reach me till April 20. 


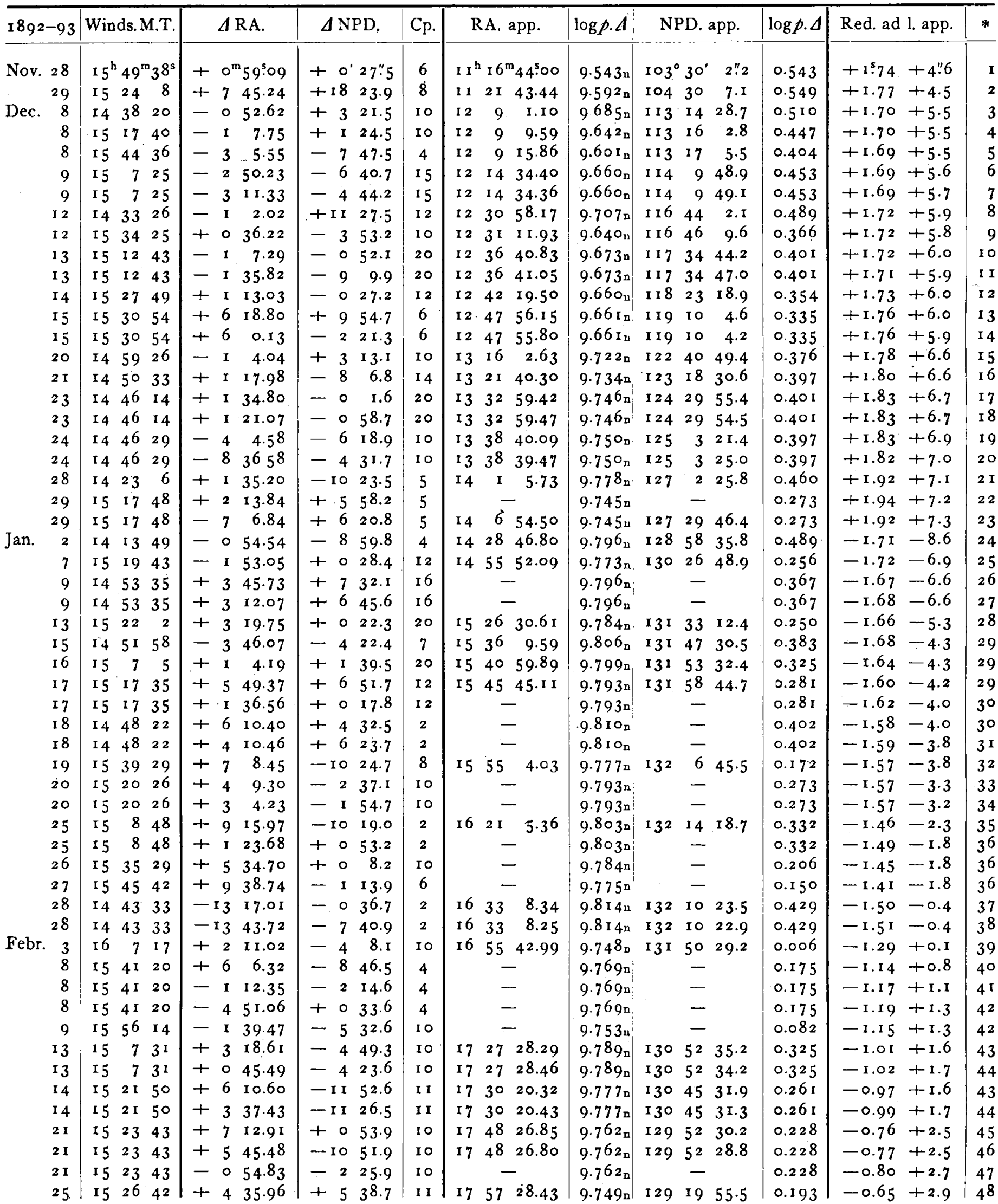




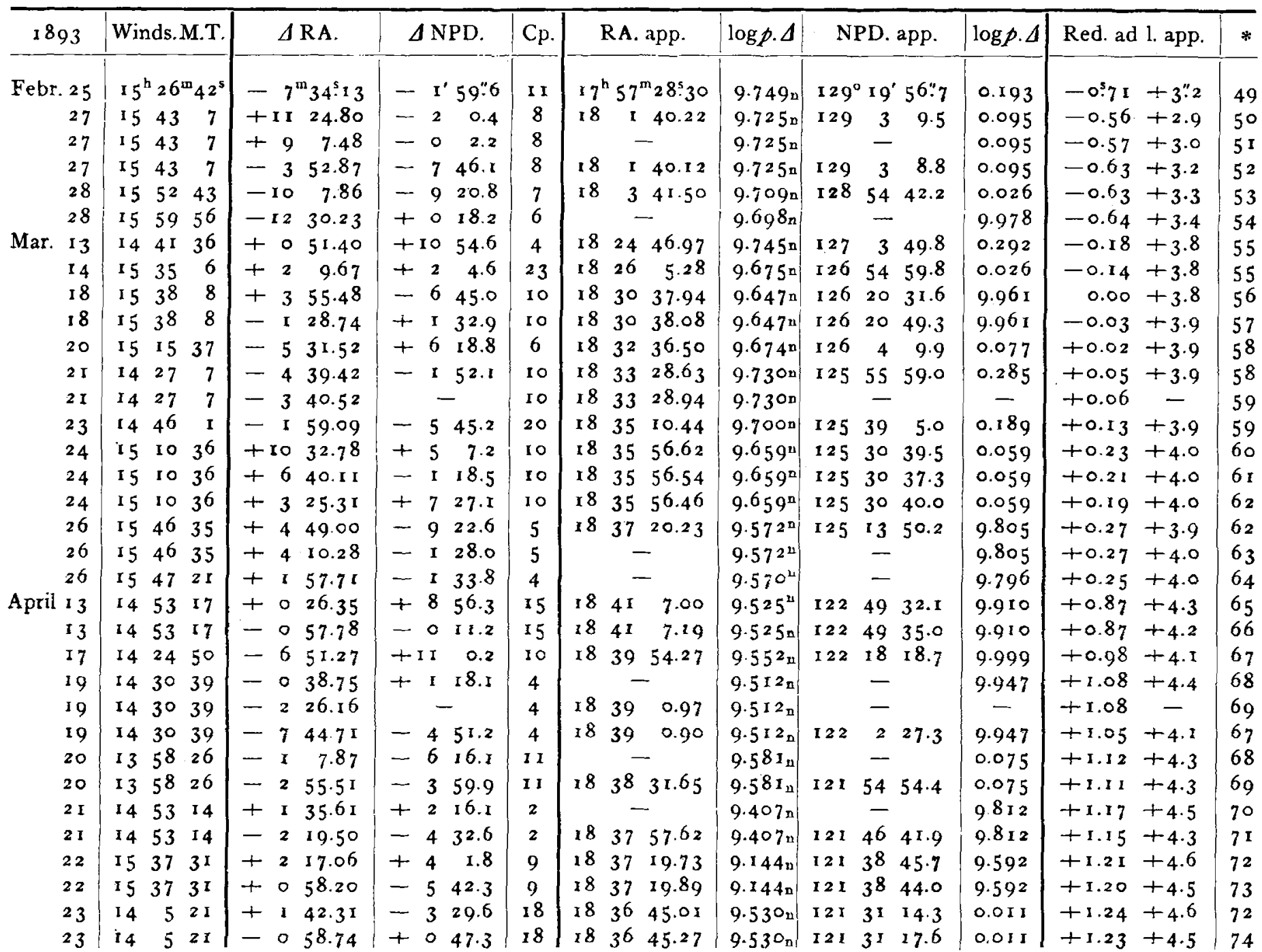

Mean Places of the Comparison Stars for the Beginaing of the Year of Observation.

\begin{tabular}{|c|c|c|c|c|}
\hline * & & RA. & NPD. & Authority \\
\hline I & $1 I^{h}$ & $15^{m} 43^{s} \cdot 17$ & $103^{\circ} 29^{\prime} 30^{\prime \prime} \mathrm{I}$ & Lal. 21639 \\
\hline 2 & 11 & I 356.43 & 104 II $3^{8.7}$ & Naut. Alm. 1892 \\
\hline 3 & I 2 & 952.02 & I I 3 I I 1 I.7 & $\mathrm{AOe}_{2} \mathrm{I}_{2024}$ \\
\hline 4 & & 1015.64 & 113 I $4 \quad 32.8$ & $\mathrm{AOe}_{2} \mathrm{I}_{2034}$ \\
\hline 5 & I 2 & $12 \quad 19.72$ & II3 $24 \quad 47.5$ & Lal. $23027, \mathrm{AOe}_{2}$ I 2058-9 \\
\hline 6 & I 2 & 1722.94 & II 4 I 624.0 & $Y_{3} 5255$, Stone 6880 \\
\hline 7 & 12 & 1744.00 & $114 \quad 14 \quad 27.6$ & $\begin{array}{c}\text { Cape Cat. }(1850) 2221, Y_{3} \\
5260 \text {, Stone } 6885\end{array}$ \\
\hline 8 & & 3158.47 & I I $6 \quad 32 \quad 28.7$ & $\begin{array}{l}\mathrm{AOe}_{2} \text { I } 2289-90, \mathrm{Y}_{3} 53^{6} 5 \\
\text { Quet. } 5 \text { I } 69 \text {, Stone } 7000, \\
\text { Io yr. I } 97\end{array}$ \\
\hline 9 & & $30 \quad 33.99$ & I I $649 \quad 57.0$ & $\begin{array}{l}\mathrm{AOe}_{2} \text { I } 2270, \text { Wash. Mur. Z }_{\text {I } 22.2 \mathrm{I}} \\
\text {. }\end{array}$ \\
\hline Io & & 3746.40 & II7 $35 \quad 30.3$ & $\begin{array}{l}\mathrm{AOe}_{2} \mathrm{I23}^{68,} \text { Wash. Mur. Z. } \\
\text { 105.123, Wash. Tr. Z. } \\
234.2\end{array}$ \\
\hline
\end{tabular}

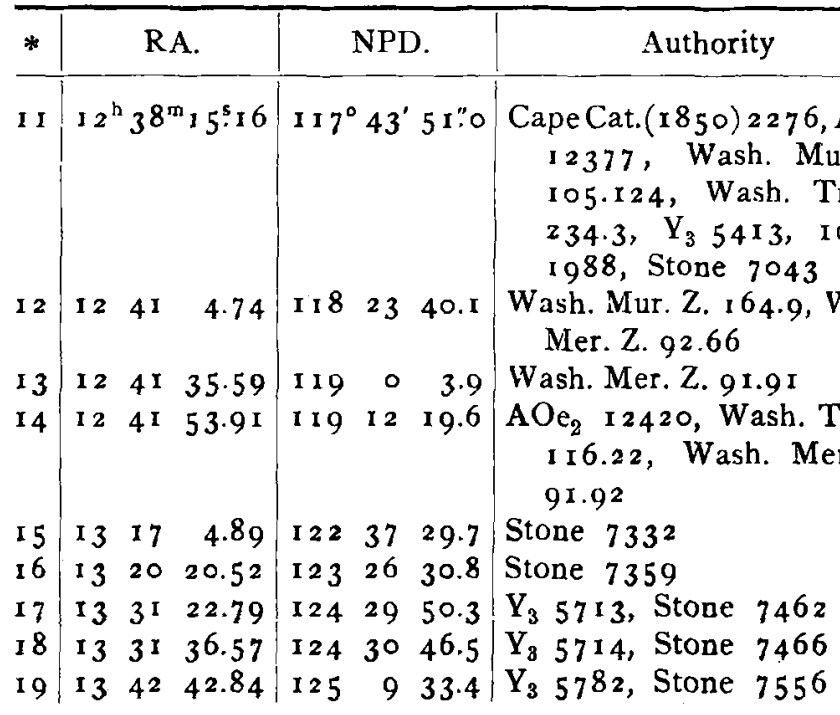




\begin{tabular}{|c|c|c|c|c|c|c|c|}
\hline$*$ & \multicolumn{3}{|c|}{ RA. } & \multicolumn{3}{|c|}{ NPD. } & Authority \\
\hline 20 & $13^{\mathrm{h}}$ & & 14.23 & $125^{\circ}$ & $7^{\prime}$ & 49.7 & $\begin{array}{l}\text { Cape Cat. }(1850) 247 \mathrm{r}, \mathrm{Y}_{3} \\
5^{829} \text {, Stone } 7604\end{array}$ \\
\hline 21 & I 3 & 59 & $28.6 \mathrm{I}$ & I 27 & 12 & 42.2 & $Y_{3} 5916$, Stone $77 \mathrm{II}$ \\
\hline 22 & I 4 & 4 & $3^{8.7}$ & × 27 & 23 & $4 \mathrm{I}$ & Star 9 mag. Equatorial \\
\hline 23 & I 4 & I 3 & $59 \cdot 42$ & I 27 & 23 & 18.3 & $Y_{3} 6016$, Stone 7821 \\
\hline 24 & 14 & 29 & 43.05 & I 29 & 7 & 44.2 & $Y_{3} 6$ I 9, Stone 7945 \\
\hline 25 & 14 & 57 & 46.86 & I 30 & 26 & 27.4 & Stone 8 I 89 \\
\hline 26 & I 5 & 2 & 27.9 & I 30 & 45 & 47 & Star $7 \frac{1}{2}$ mag. Equatorial \\
\hline 27 & I 5 & 3 & I. 6 & 130 & 46 & 37 & Star $71 / 2$ mag. Equatorial \\
\hline 28 & I 5 & 23 & $12.5^{2}$ & $x^{I}$ & $3^{2}$ & $55 \cdot 4$ & Stone 842 I \\
\hline 29 & I 5 & 39 & $57 \cdot 34$ & I 3 I & $5 \mathrm{I}$ & 57.2 & Stone 8564 \\
\hline 30 & r 5 & 44 & ro.3 & I 3 I & 58 & 57 & Star 9 mag. Equatorial \\
\hline 31 & I 5 & 46 & 10.1 & r3 $\mathbf{I}$ & 57 & 6 & Star 9 mag. Equatorial \\
\hline $3^{2}$ & I 5 & 47 & 57.15 & 132 & 17 & 14.0 & Stone 8635 \\
\hline 33 & I 5 & 55 & 23.7 & 132 & I I & 50 & Star 9 mag. Equatorial \\
\hline 34 & 15 & $5^{6}$ & 28.7 & I 32 & I I & 20 & Star 9 mag. Equatorial \\
\hline 35 & I 6 & I I & 50.85 & 132 & 24 & 40.0 & Stone 8860 \\
\hline $3^{6}$ & 16 & I 9 & 42.7 & 132 & I 3 & 28 & Star $9 \frac{1}{2}$ mag. Equatorial \\
\hline 37 & 16 & 46 & 26.85 & I 32 & I I & 0.6 & $\begin{array}{l}\text { Cape Cat. (1850) 3129, } \\
\text { Stone } 9 \text { r6o }\end{array}$ \\
\hline $3^{8}$ & 16 & 46 & 53.48 & 132 & 18 & 4.2 & $\begin{array}{l}\text { Cape Cat. (1850) } 3 \times 35 \text {, } \\
\text { Stone } 9169\end{array}$ \\
\hline 39 & 16 & 53 & 33.26 & I 3 I & 54 & 37.2 & Stone 9242 \\
\hline 40 & 17 & 6 & I 5.1 & I $3 \mathbf{I}$ & 33 & 48 & Star 9 mag. Equatorial \\
\hline $4 \pi$ & 17 & I 3 & $33 \cdot 5$ & $13^{x}$ & 27 & $x 6$ & Star $91 / 2$ mag. Equatorial \\
\hline 42 & 17 & 17 & 12.1 & I 3 I & 24 & 28 & Star 9 mag. Equatorial \\
\hline 43 & I $\eta$ & 24 & 10.69 & I 30 & 57 & 22.9 & Stone 9537 \\
\hline 44 & 17 & 26 & 43.99 & 130 & $5^{6}$ & 56.1 & Stone 9565 \\
\hline 45 & 17 & $4^{I}$ & 14.70 & 129 & $5^{1}$ & 338 & Stone 9683 \\
\hline 46 & 17 & 42 & 42.09 & I 30 & 3 & I 8.2 & $\begin{array}{l}\text { Cape Cat. (1850) 3388, } \\
\text { Stone } 9707\end{array}$ \\
\hline 47 & I 7 & 49 & 22.1 & 129 & 54 & $5^{\mathrm{I}}$ & Star $81 / 2$ mag. Equatorial \\
\hline 48 & I 7 & $5^{2}$ & 53.12 & I 29 & r 4 & I 3.9 & Stone 9798 \\
\hline
\end{tabular}

\begin{tabular}{|c|c|c|c|c|c|c|c|}
\hline * & \multicolumn{3}{|c|}{ RA. } & \multicolumn{3}{|c|}{ NPD. } & Authority \\
\hline 49 & $18^{\mathrm{h}}$ & & 3.14 & $129^{\circ}$ & $2 I^{\prime}$ & $53 ! \mathrm{r}$ & Stone 9904 \\
\hline $5^{\circ}$ & 17 & $5^{\circ}$ & I 5.98 & 129 & 5 & 7.0 & Stone 9772 \\
\hline 51 & I 7 & $5^{2}$ & 33.2 & I 29 & 3 & 9 & Star 8 mag. Equatorial \\
\hline 52 & 18 & 5 & 33.62 & I 29 & IO & $5^{1.7}$ & Stone 9908 \\
\hline 53 & 18 & 13 & 49.99 & I 29 & 3 & $59 \cdot 7$ & Stone $999^{\circ}$ \\
\hline 54 & I 8 & 16 & I $3 \circ$ & I 28 & 54 & 22 & Star $81 / 2$ mag. Equatorial \\
\hline 55 & 18 & 23 & $55 \cdot 75$ & I 26 & $5^{2}$ & 51.4 & $Y_{3} 7982$, Stone 10078 \\
\hline 56 & 1 8 & 26 & 42.46 & I 26 & 27 & I 2.8 & Wash. Mur. Z. $49 \cdot 3$ \\
\hline 57 & I 8 & 32 & 6.85 & 126 & 19 & I 2.5 & Wash. Mur. Z. 49.5 \\
\hline 58 & I 8 & 38 & 8.00 & I 25 & 57 & 47.2 & $Y_{3} 8087$, Stone 10194 \\
\hline 59 & I 8 & 37 & 9.40 & 125 & 44 & $46 \cdot 3$ & $\begin{array}{l}\text { Sydney Cat. I } 859, \text { Melb }_{2} \\
934 \text {, Stone } 10183\end{array}$ \\
\hline 60 & 18 & 25 & $23.6 \mathrm{r}$ & I 25 & 25 & 28.3 & $\begin{array}{l}\text { Wash.Tr.Z. } 44.47, Y_{3} 7993 \text {, } \\
\text { Stone } 10086\end{array}$ \\
\hline $6 \mathrm{I}$ & 18 & 29 & I 6.22 & I 25 & $3 \mathbf{r}$ & $5+8$ & Wash. Tr. Z. 44.48 \\
\hline 62 & 18 & 32 & 30.96 & 125 & 2.3 & 8.9 & Wash. Tr. Z. 44.49 \\
\hline 63 & 18 & 33 & 9.4 & 125 & 15 & 14 & Star $81 / 2$ mag. Equatorial \\
\hline 64 & 18 & 35 & 22.0 & 125 & 15 & 19 & Star 9 mag. Equatorial \\
\hline 65 & 18 & 40 & 39.78 & 122 & 40 & $3^{1 \cdot 5}$ & Wash. Mur. Z. 25.77 \\
\hline 66 & 18 & 42 & 4.10 & 122 & 49 & 42.0 & $\begin{array}{l}\text { Wash. Mur. Z. } 25 \cdot 78 \text {, Cape } \\
\text { Cat. (1 } 850) 3668 \text {, Stone } \\
\text { I0228 }\end{array}$ \\
\hline 67 & I 8 & 46 & $44 \cdot 56$ & I 22 & 7 & 14.4 & $\begin{array}{l}\text { Wash. Mur. Z. } 25 \cdot 79 \text {, Cape } \\
\text { Cat. (1850) } 3692 \text {, Stone } \\
\text { I0275 }\end{array}$ \\
\hline 68 & I 8 & 39 & 38.4 & 122 & $\mathbf{I}$ & 5 & Star 8 mag. Equatorial \\
\hline 69 & 18 & $4 I$ & 26.05 & I 21 & 58 & 50.0 & Wash, Tr. Z. 30.79 \\
\hline 70 & I 8 & 36 & 20.6 & 121 & 44 & 22 & Star 9 mag. Equatorial \\
\hline $7 \mathrm{I}$ & 18 & 40 & I 5.97 & I 2 I & $5^{I}$ & ro.2 & Wash. Tr. Z. 30.78 \\
\hline 72 & r 8 & 35 & I. 46 & I 2 I & 34 & $39 \cdot 3$ & $\begin{array}{l}\text { Wash. Mur. Z. 39.24, } \mathrm{Y}_{3} \\
\quad 8063\end{array}$ \\
\hline 73 & 1 8 & $3^{6}$ & 20.49 & 121 & 44 & $2 \mathrm{I} .8$ & $Y_{3} 8072$ \\
\hline 74. & 18 & 37 & 42.78 & I 2 I & 30 & 25.8 & Wash. Mur. Z. 39.26 \\
\hline
\end{tabular}

Private Observatory, The Peninsula, Windsor, N. S. Wales, I 893 May 5.

Fohn Tebbutt.

\section{Ueber die leuchtenden Nachtwolken.}

In der Nacht vom 8. zum 9. Juli d. J. sind die leuchtenden Nachtwolken an mehreren Orten in Norddeutschland zum ersten Male in diesem Jahre gesehen worden.*) Kurz vor $101 / 2^{\text {h }}$ Abends Ortszeit sah ich sie von Steglitz aus am NW-Himmel. Das Licht derselben war aber sehr schwach, so dass dasselbe sich kaum von dem Dämmerungshimmel abhob. Um $\mathrm{II}^{\mathrm{h}}$ war das Phänomen yerschwunden. Nach Mitternacht gegen $14^{\mathrm{h}}$ sah ich dagegen die leuchtenden Nachtwolken an der Nordseite des Himmels bis zu einer Höhe von etwa $5^{\circ}$ recht hell. Herr F.S. Archenhold,
Sternwarte Grunewald bei Berlin, hat das Phänomen ebenfalls beobachtet; auch in Hamm bei Hamburg ist nach einer brieflichen Mittheilung dasselbe gegen ${ }_{12} 1 / 4^{b}$ gesehen worden.

Bemerkenswerth erscheint auch hierbei die Thatsache, auf welche bereits in A. N. 3 r20 aufmerksam gemacht worden ist, dass das Phänomen Nachmitternachts wesentlich stärker entwickelt ist als Vormitternachts, wie es auch Nachmitternachts viel häufiger beobachtet worden ist als Vormitternachts.

Berlin, Sternwarte, I 893 Juli Io.

O. Fesse.

*) In Kiel waren ebenfalls am 8. Juli die leuchtenden Nachtwolken sehr aufällig. Prof. E. Lamp hat dieselben bereits am 15 . und r6. Juni $\mathbf{I}^{\text {h }}$ Abends bemerkt. $\boldsymbol{K r}$. 